\title{
On the Application of Current Commutations Rules to Nonleptonic Decays.
}

\author{
R. NATAF \\ Institut de Physique Nucléaire, Division de Physique Théorique - Orsay \\ (Nuovo Cimento, $52 \mathrm{~A}, 7(\mathbf{1 9 6 7 )})$
}

On p. 15, please substitute lines 13 to 19 with the following text:

Actually, to be consistent, we had to take the Hamiltonian in the Ileisenberg picture. Now:

$$
{ }_{\text {out }}\langle\alpha \pi \mid \beta\rangle_{\text {in }}={ }_{\text {out }}\langle\alpha \pi|S| \beta\rangle_{\text {out }},
$$

$S$ being the scattering matrix for all the interactions. But, to first order in the weak interactions, we can write

$$
{ }_{\text {out }}\langle\alpha \pi|S| \beta\rangle_{\text {out }}={ }_{\text {out }}\left\langle\alpha \pi\left|S_{\text {weak }}\right| \beta\right\rangle_{\text {盀 }}
$$

where the in (and out) states are defined with the weak interactions being switched off at any time.

$$
S_{\text {weak }}=1-i \int \mathrm{d}^{4} x \mathscr{H}_{w}(x)
$$

with $\mathscr{H}_{w}(x)$ in the Heisenberg picture for the Wightman fields (all the interactions being switched off at $t= \pm \infty$ : this corresponds to the interaction picture for the Dyson fields). 